\title{
Simple Genetic Tools to Study Fruiting Body Development in Fungi
}

\author{
Philippe Silar ${ }^{*}$
}

\section{Univ Paris Diderot, Sorbonne Paris Cité, Laboratoire Interdisciplinaire des Energies de Demain, 75205 Paris CEDEX 13 France}

\begin{abstract}
Unlike for animal and plant development, the molecular processes involved in shaping the multicellular fruiting bodies of fungi are almost unknown. Especially, the interplay between the mycelium, the maternal tissues and zygotic ones are seldom investigated. Here, I summarized simple genetic methods that permit to allocate site(s) of action for genes whose mutations block fruiting body development. These involve the formation of genetic mosaics and grafting, as used for many decades to study embryo development in animals and plants. They are easily implemented without the requirement of complex equipment. Yet, they provide useful information when one wants to order genes into pathways acting during fruiting body production. Examples taken from the study of the model ascomycete Podospora anserina illustrate how they can be interpreted.
\end{abstract}

Keywords: Genetic mosaics, grafting, multicellular fruiting body, mutant, perithecium, Podospora anserine, sporophore development.

\section{INTRODUCTION}

The "higher" fungi (i.e., the Ascomycota and Basidiomycota) are able to differentiate complex multicellular structures to ensure efficient dispersal (Fig. 1). Yet, unlike animals and plants, in which the molecular mechanisms involved in shaping the multicellular structures are now well-known, the pathways involved in building fungal fruiting bodies remained to be elucidated. Though numerous mutants lacking mature fruiting bodies are available [1,2], few have been studied up to the point where the actual function during development of the mutated genes they contain has been determined. Simple genetic techniques similar to those used to study development in animals and plants, namely genetic grafting and mosaic analyses, can also be used in fungi to study the building of sporophores. The aim of this paper is to present these methods and what can be deduced from the results. Examples will mostly be taken from my favorite fungus $P$. anserina, a coprophilous ascomycete that is well-suited to study the development of the fruiting body. A great advantage is that its complete life cycle takes only seven days and the maturation of fruiting body from fertilization to ascospore discharge lasts four days (for a review on the biology and genetics of $P$. anserina see [3]). The $P$. anserina genome sequence is available $[4,5]$ and methods to rapidly inactivate or overexpress genes have been designed [3]. Classical genetic studies provided numerous mutants affecting fruiting body development [6, 7]. This organism is thus perfectly suited for extensive analysis of fruiting body development using molecular genetics (see for example [8, 9]). In this species,

*Address correspondence to this author at the Univ Paris Diderot, Sorbonne Paris Cité, Laboratoire Interdisciplinaire des Energies de Demain, case courrier 7040 Lamarck, 75205 Paris CEDEX 13 France;

Tel: 331572784 72; E-mail: philippe.silar@univ-paris-diderot.fr the fruiting body (Fig. 1H) is a small flask-shape structure called a perithecium, which matures after the fertilization of the female gamete (ascogonium) by a male one (spermatium). In $P$. anserina, the ascogonium is rapidly surrounded by maternal hyphae for protection, often before fertilization. The resulting female gametangium is called protoperithecium and provides haploid maternal nuclei and the cytoplasm for the zygotic tissue, including mitochondria. The male gamete provides during fertilization a single paternal nucleus. In $P$. anserina, a fruiting body always originates from a single fertilization event. However, the male nucleus divides in the fertilized gametangium to yield a multinucleated cell with many nuclei of either paternal or maternal origins. From this multinucleated cell, paternal/maternal pairs of nuclei enter a lineage that will ultimately produce the asci. After fertilization, further maturation of the maternal tissue will give rise to the peridium of the fruiting body as well as the sterile tissue of the centrum (e.g., the nourishing cells located at the bottom of the ascus and the paraphyses), which is embedded with the zygotic one that results from fertilization. This type of development is called ascohymenial and is typical of the majority of filamentous ascomycetes (i.e., all of them except the Dothideomycetes and a few other species). Analysis may be somewhat different in ascolocular ascomycetes (e.g., the Dothideomycetes for which the fruiting body develops before the gametes) and the Basidiomycota (for which there are no maternal/paternal progenitors). However, the same methods can be applied with these fungi and will yield similar results.

P. anserina possesses two mating types, mat+ and mat-, and fertilization occurs only between mat+ and matgametes. This ensures that the time of fertilization can be chosen and that maturation of fruiting bodies can be synchronized. It is also possible to choose which partner will act as female and which will act as male. Indeed, spermatia 

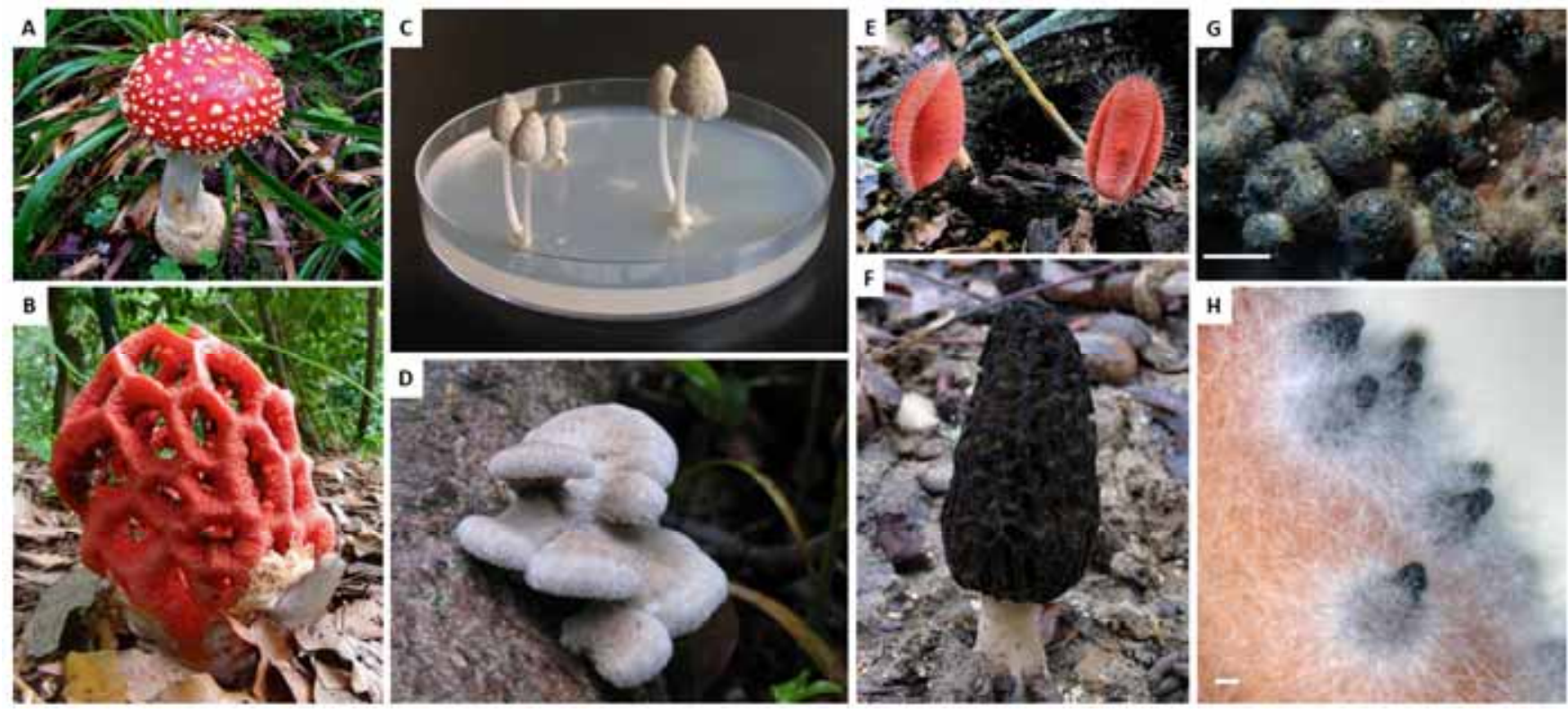

Fig. (1). Diversity of fruiting bodies in fungi.

Basidiomycetes usually differentiate large sporophores (carpophores), some of which can adopt extraordinary complex forms (A: Amanita muscaria and B: Clathrus ruber). The models most frequently used to study fruiting body development in Basidiomycota are Coprinopsis cinerea and related species (C: Coprinopsis sp. and D: Schizophyllum commune). While ascomycetes may differentiate large sporophores no less extraordinary than those of basidiomycetes such as E: Cookeina sp.; F: Morchella conica, most species differentiate minute fruiting bodies (G: Emericella (=Aspergillus) nidulans and $\mathbf{H}$ : Podospora anserina). Several of these species that are easily cultivated and readily producing fruiting bodies in the laboratory are used to study development through genetic analysis of mutants. Scale bar: $200 \mu \mathrm{m}$.

are easily dislodged by water, whereas ascogonia are tightly bound to the mycelium. Supernatants of Petri plates flooded with water contain only spermatia that can be used to fertilize ascogonia, simply by pouring them onto mycelia of opposite mating type. Many $P$. anserina mutants affecting perithecium development are available. Like sterile mutants of other fungi, they are potentially blocked at many stages of fruiting body production or maturation. Firstly, the mycelium may be unable to initiate perithecium development, as maturation likely requires nutrients and energy that is provided through underlying hyphae. An unhealthy mycelium (e.g., with a defects in amino acid biosynthesis, protein polymerization, respiration, etc.) often results in sterility. Secondly, gametes may not be properly differentiated. Thirdly, fertilization may be blocked before or after gamete recognition. Finally, maturation of the fruiting body may be impaired either because the zygotic tissue is impaired or because the maternal tissue is compromised. Microscopic examination can determine whether gametes are differentiated or not, but is of little help in determining whether defects in the mycelium, the maternal or the zygotic tissues are responsible for sterility. The methods discussed here may be used to determine at which step development is impaired. Importantly, they do not require complex tools to be set up.

\section{GRAFTING ANALYSIS}

Surgical interventions on fruiting body, explants of tissues from developing fructifications or grafting have seldom been applied to analyze fungal fruiting body development. Bourne et al. reviewed about 20 years ago what has been done with basidiomycetes carpophores (the large sexual fruiting bodies of macromycetes, Figs. 1A-F) [10]. Since then, nothing appears to have been published on the subject. The primary interest was to understand renewed fruiting (the fact that fragments of carpophores yield new crops of fructifications faster than mycelia), transfers of nutrients within the fruiting bodies or the commitment of particular tissue of the fruiting bodies to complete differentiation. More recently, I showed that $P$. anserina whole perithecia can be grafted onto self and non-self mycelia [11]. This is very easy to do. One only has to lift gently with a needle a developing fruiting body, along with the smallest amount of underlying hyphae, and put the graft onto another mycelium. Note that, for $P$. anserina, injured fruiting bodies stop their maturation, showing that grafting parts of perithecia together would not result in proper development. However, it is highly likely that grafting of whole fruiting bodies can be implemented in many species and in some of them grafting together pieces of fruiting body may prove successful (e.g., in Pezizomycetes, where fruiting bodies appear less constrained).

Grafting enables following transfer of materials and resources from the mycelium into the maturing fruiting body. For example, the transfer of mitochondrial proteins to two-day old perithecia grafted onto a mycelium producing a GFP targeted to the mitochondria, mito-GFP [12] is depicted in (Fig. 2). As previously described for the su12-GFP protein [11], which labels functional ribosomes [13], transfer of mito-GFP can be observed in some of the 
A

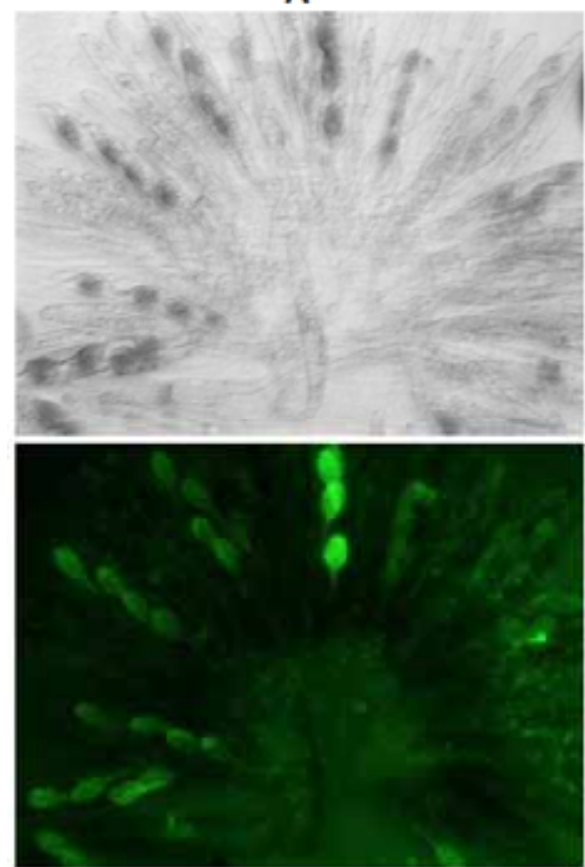

B

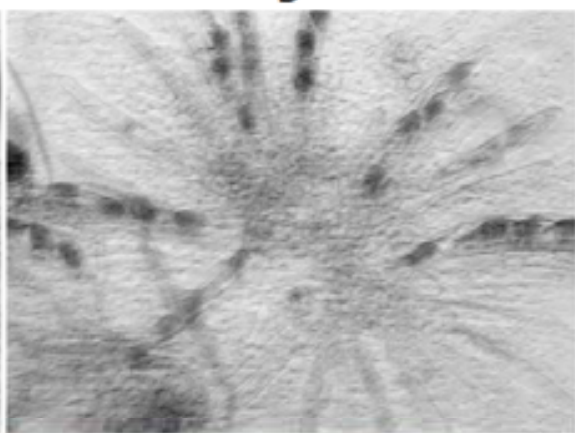

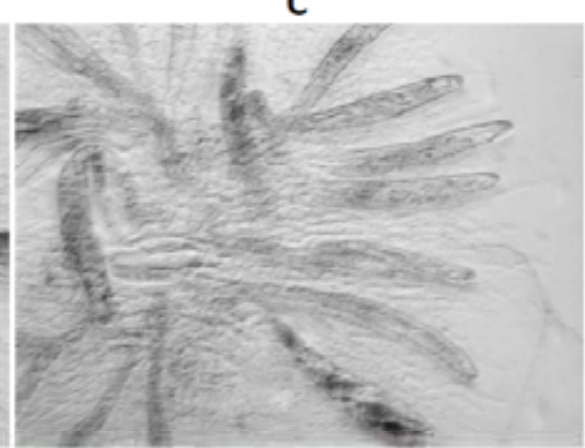
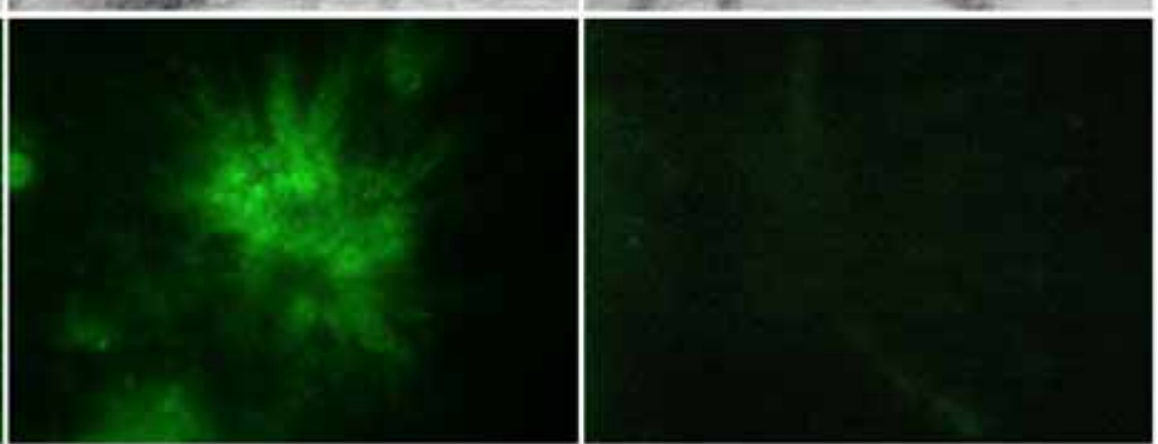

Fig. (2). Entry of mito-GFP into the fruiting body.

Mito-GFP is a chimaeric protein composed of a mitochondrial targeting sequence fused to the eGFP coding sequence and controlled by the $P$. anserina constitutive $g p d$ promoter [12]. (A) In a cross where both parents express the protein, fluorescence can be detected in all tissues of the centrum, including asci where fluorescence is intense. (B) When a 2-day old wild-type perithecium (lacking the protein) is grafted onto a mycelium expressing mito-GFP, the protein is transferred to the nourishing cells located at the bottom of the centrum, but not to the asci or paraphyses. (C) If the grafting is made on a het-s mycelium having vegetative incompatibility with the grafted perithecia taken from a het-s mycelium, no mito-GFP is transferred, although the fruiting bodies continue their development and ascospores are produced.

cells that are of maternal origin (e.g., nourishing cells and, not shown on the Fig. 2, cells from the peridium), but not in zygotic cells such as asci. Nevertheless, apart from tracing components entering (or exiting) the fruiting body, grafting experiments may also give some answers regarding the role of genes in the mycelium versus in the fruiting body during development (Fig. 3). Indeed, if after grafting wild-type fructifications onto mutant mycelia, the maturation is arrested, this argues that the mutant gene is necessary at least in the mycelium, while if development continues the gene is dispensable. Similarly, if mutant fruiting body can be obtained (e.g., for mutants arrested late in the maturation process), grafting them onto wild-type mycelium, should answer whether the mutant gene is required in the fruiting body or not. In this way, we have studied the role of the genes IDCl (coding a large protein of unknown function), PaMpkl and PaMpk2 (both coding for MAP kinases), as well as PaNoxl (coding a catalytic subunit of a NADPH oxidase) and PaNoxR (coding a cytosolic regulator of PaNox 1). All these mutants do not form any fruiting body, although they differentiate male and female gametes [11, 14]. We also studied a mutant of PaAlr1, which codes for a magnesium transporter. The $P a A l r l^{\Delta}$ mutant is arrested at the precise stage where asci are formed, but ascospores still contain only two nuclei, i.e., just after the delimitation of the ascospores [4]. Indeed, in $P$. anserina, ascospores are delimited around two nuclei, which further divide when the ascospores mature [15]. A typical mature ascospore contains around 30 nuclei. We found that PaMpk1, PaMpk2 and IDC1 are required in the mycelium, while PaNoxl, PaNoxR and PaAlrl are not. Moreover, $\mathrm{PaAlr}{ }^{\Delta}$ perithecia grafted onto wild-type mycelium still arrest their development, showing that PaAlr1 act autonomously in perithecia (Fig. 3 and [4]). We also analyzed the defect in the shape of perithecia of the $\Delta p a h 1$ mutant affected in the homeodomain transcription factor pah1 ( $\triangle p a h 1$ perithecia are more roundish and covered with shorter hairs), as well as the lack of beak in the $\Delta p a h 2$ and $\Delta p a h 5$ mutants, affected in the pah2 and pah5 homeodomain transcription factors respectively [8]. The beak is the dark structure visible in (Fig. 1H) at the top of the fruiting body. It contains an ostiole that allows ascospore discharge. In the three cases, grafting did not eliminate the defects, showing that the homeobox genes act autonomously in the maturing fruiting bodies.

Alone, grafting experiments are not sufficient to provide all the answers regarding the role of the mycelium $v s$ the fruiting body, but combined with the mosaic experiments explained below, a good idea of the role of the gene can be gathered.

\section{MOSAIC ANALYSIS WITH AUTONOMOUS PIGMENT-LESS MUTANTS}

Another simple way to assess the involvement of the maternal/zygotic tissue of the fruiting body versus the 

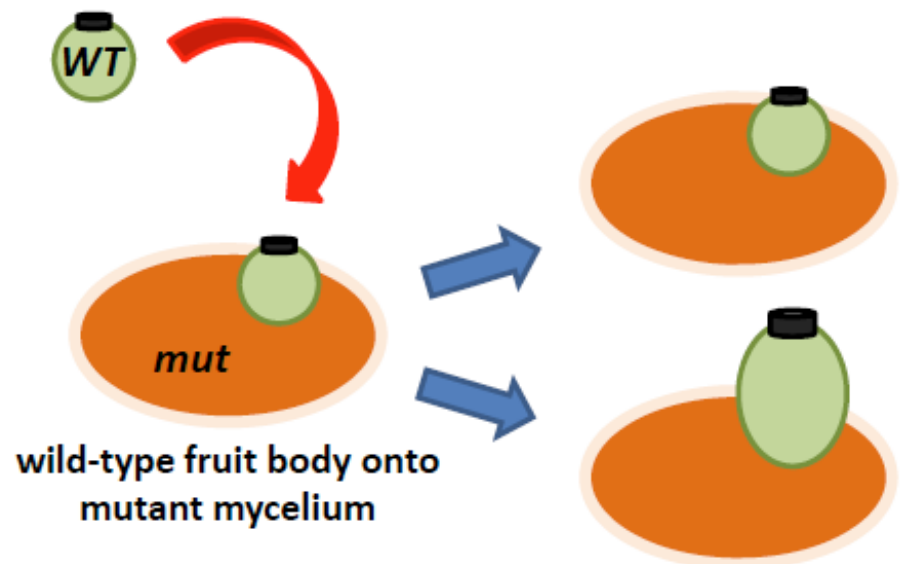

\author{
maturation stops: \\ the gene altered in the mutant is \\ required in the mycelium
}

\title{
maturation continues: the gene altered in the mutant is NOT required in the mycelium
}
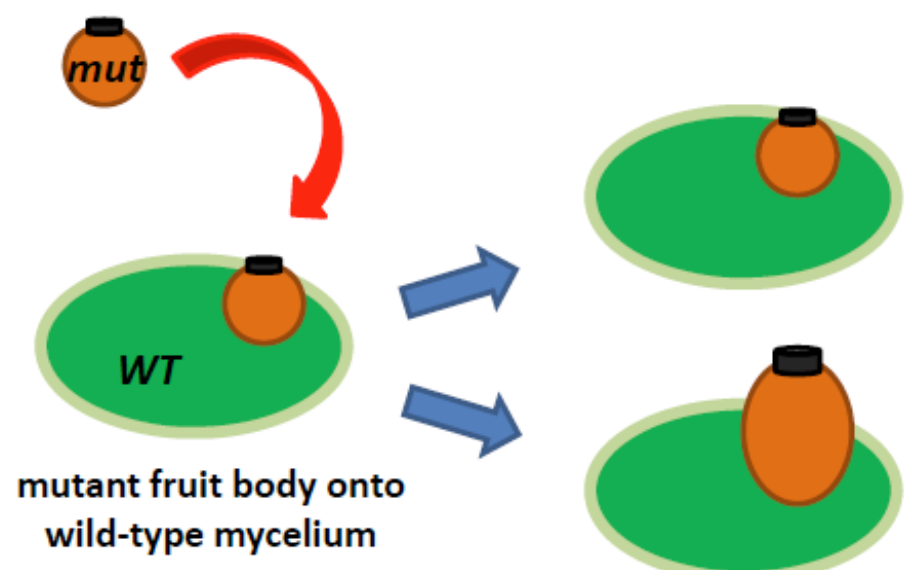

maturation stops: the gene altered in the mutant is required in the perithecium

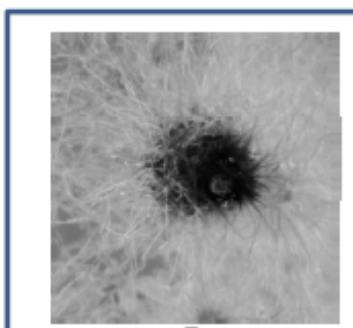

WT $\rightarrow$ WT
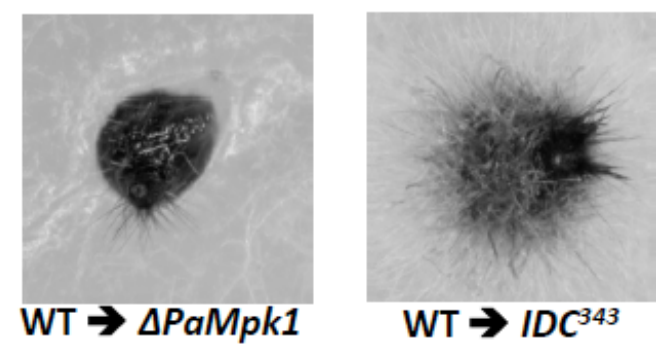

$\mathrm{WT} \rightarrow I D C^{343}$

maturation continues: the gene altered in the mutant is NOT required in the perithecium

Fig. (3). Grafting with mutants.

At the top is depicted all the grafting combinations and outcomes possible between wild type and mutants. At the bottom left are shown results of grafting wild-type perithecia onto various recipient mycelia. To this end, perithecia obtained two days after fertilization were lifted with a needle from the donor mycelium, taking as less neighboring hyphae as possible, and gently deposited on the recipient one. They were then incubated two more days before the pictures were taken. Grafting onto the $\triangle P a M p k 1$ mutant arrests maturation (as seen by the lack of hair - setae - on the graft), while grafting onto the $I D C^{343}$ mutant affected in the PaNoxl gene does not. From these data it can be concluded that the PaMpkl gene is likely required in the mycelium, while PaNoxl is likely not. At the bottom right is a rosette of asci of PaAlrl ${ }^{4}$ perithecium grafted onto a wild-type mycelium. All asci have arrested their maturation (no darkly-colored ascospore is visible) showing that PaAlrl is required in the fruiting body for proper development.

mycelium is through the analysis of genetic mosaics with pigment-less mutants [16]. Such mosaics are easily obtained by mixing together hyphae from both partners in a microtube with a pestle or an apparatus such as a Fastprep (MP Biomedicals). For this analysis, the color-less mutations must be autonomous, meaning that pigments from neighboring wild-type cells will not diffuse into the mutant ones when mixed together. This allows tracing in mature perithecia the origin (either wild-type or mutant) of the cells. When mixed heterokaryons of wild type and pigment-less mutant are incubated, three kinds of perithecia may be produced: those with only dark cells, those with only pigment-less cells and mosaic ones containing both kinds of cells. In $P$. anserina, we routinely use the PaPks 1-193 mutant lacking the polyketide synthase that acts at the first step of melanin biosynthesis [17] in association with developmental mutants arrested early during development (Fig. 4). In the conditions we used, mosaic perithecia are rare, indicating that few initial nuclei participate in the formation of the maternal tissues of the fruiting bodies. Depending upon the mode of action of the gene, we obtain either a mixture of dark and light perithecia or only light perithecia. In the former case, dark perithecia can only be made up from mutant hyphae (as the mutant does not contain 

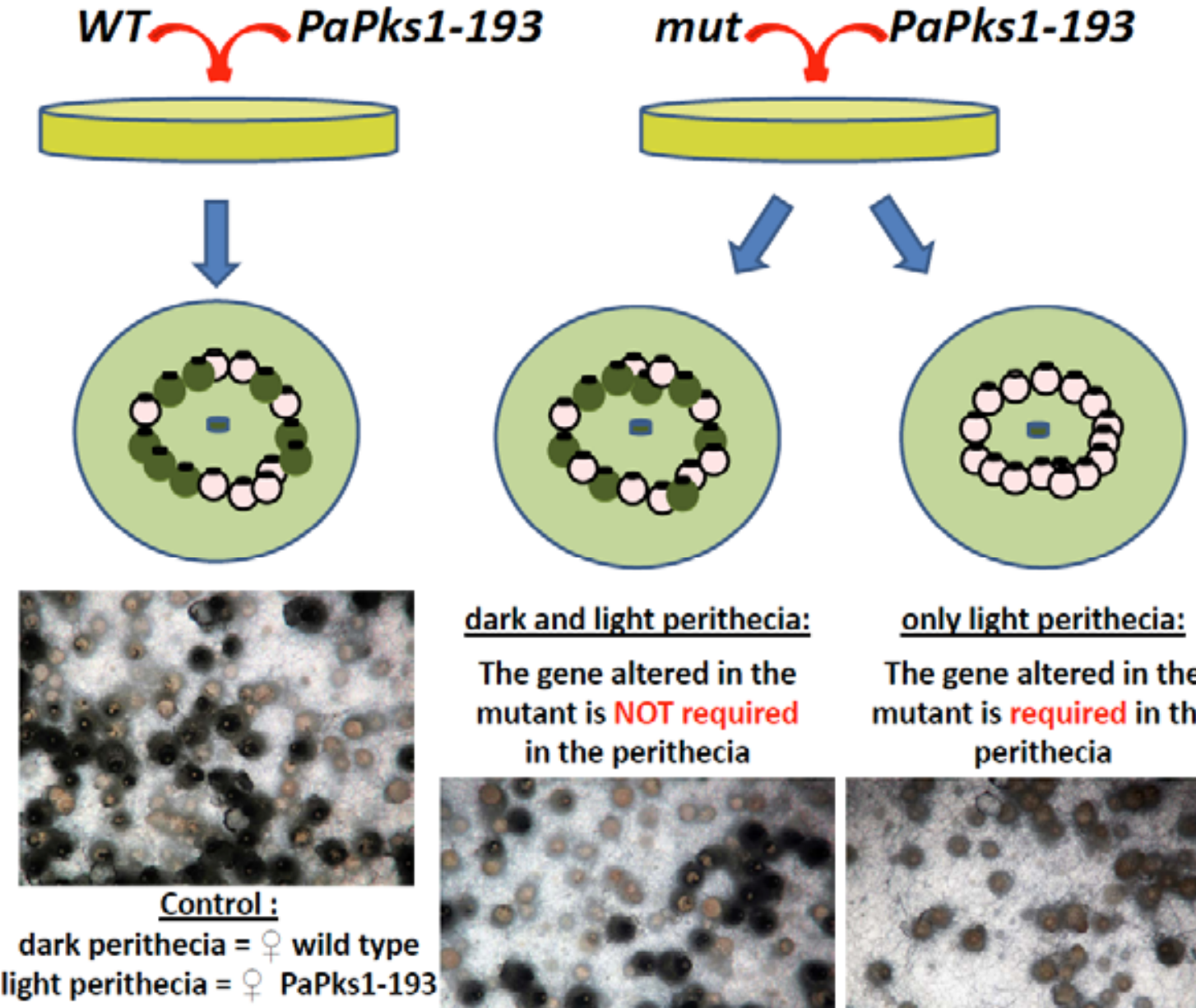

dark and light perithecia: The gene altered in the mutant is NOT required in the perithecia

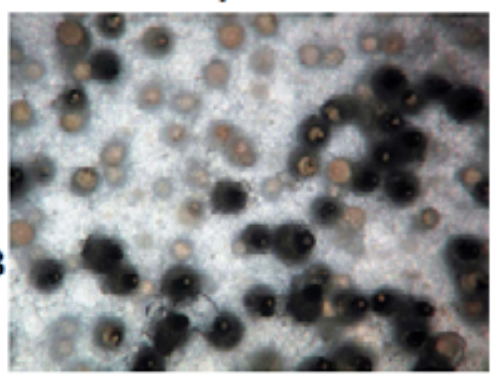

$\triangle P a M p k 1$ x PaPks1-193 only light perithecia:

The gene altered in the mutant is required in the perithecia

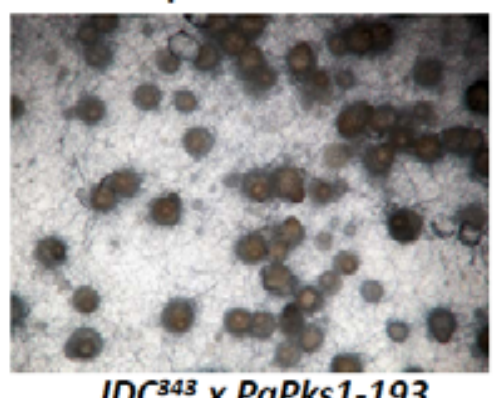

Fig. (4). Mosaic analysis with the PaPks1-193 mutant.

Mosaics were made by mixing together in $500 \mu \mathrm{L}$ sterile water $1 \mathrm{~mm}^{3}$ explants of the desired mycelia taken from fresh stock plates using a Fastprep (MP Biomedicals, Santa Ana, CA USA) at speed 4.0 during 20 seconds. Plates were inoculated at the center with $5 \mu$ l of the mixtures. See text for detailed explanation of the outcomes. Bottom pictures were obtained after seven days of incubation.

the PaPks1-193 mutation) and hence the mutated gene is not required inside the perithecia. Its most likely place of action is therefore the mycelium. On the contrary, recovery of only light perithecia show that perithecia containing mutant cells cannot mature and therefore that the mutated gene is required inside the fruiting body. Note that recovery of high numbers of mosaic perithecia would indicate a non-autonomous action of the mutated gene, i.e., only a fraction of the cells from the fruiting bodies being wild-type would be sufficient to promote normal development, possibly because the product of the wild-type gene would diffuse in the whole fruiting body. We have used this method to analyze mutants of the PaNox1, PaNoxR, PaMpk1, PaMpk2 and IDC1 genes $[14,16,18,19]$. The results confirmed and completed those of the grafting analysis: PaMpk1 and PaMpk2 are not required in the perithecium, but only in the mycelium; PaNoxl and PaNoxR are required only in the fruiting body and $I D C 1$ is required in both the mycelium and the fruiting body.

Mosaics can be implemented in many other fungi. The first use of such analysis was made in Neurospora crassa, in which a per-1 mutant affected in fruiting body pigmentation has been used to trace the origin of the different parts of the perithecium [20]. In these early analyses, no developmental mutant was involved. Later, the biAl and $c l 3$ mutants were used for a similar analysis of cleistothecium ontogeny in Aspergillus nidulans [21]. This species is homothallic and fruiting bodies can results from self or non-self fertilizations. biAl confers in an autonomous manner a blue color to the cleistothecium wall and $c l 3$ fruiting bodies lack color, though both mutants act non-autonomously on ascospore color, i.e., ascospores have the color of the cleistothecium wall irrespective of their genotype [22]. These mutants were thus used to follow whether fertilization are self or non-self and to estimate the number of nuclei at the origin of cleistothecia (the estimated number was two nuclei). However, more recently, Bruggeman et al. [23] used biA1 and clB6 (another colorless mutant), along with mitochondrial markers to analyze vegetatively-compatible and vegetatively-incompatible crosses. They show that it is really possible to trace the paternal or maternal origin of the genetic constituents of cleistothecia only in vegetatively- 


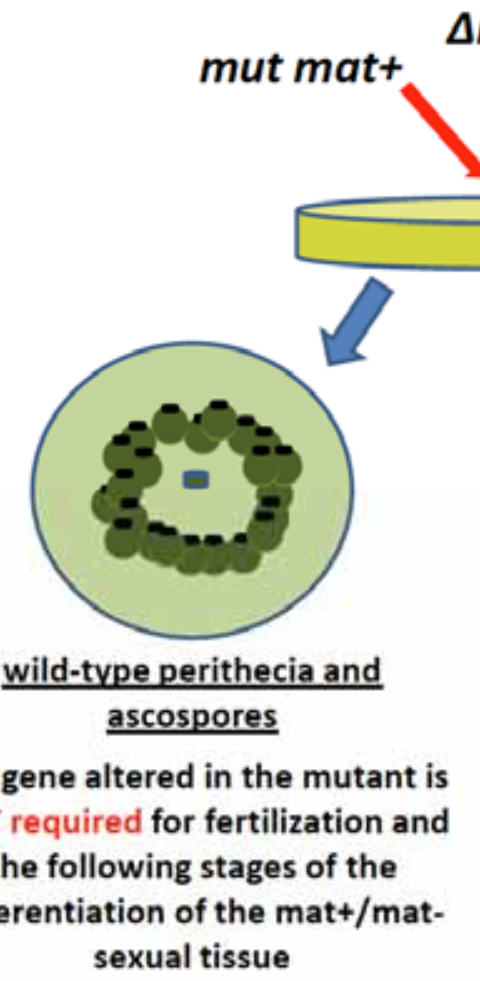

$\Delta m a t$

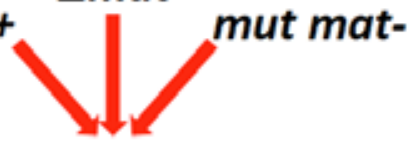

r

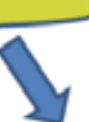

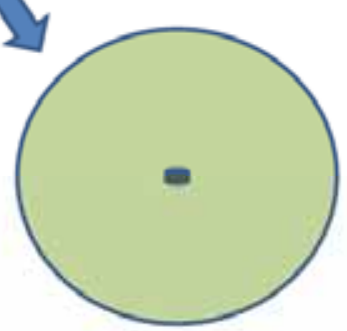

no or mutant perithecia

The gene altered in the mutant is required for fertilization or the following stages of the

differentiation of the mat+/matsexual tissue

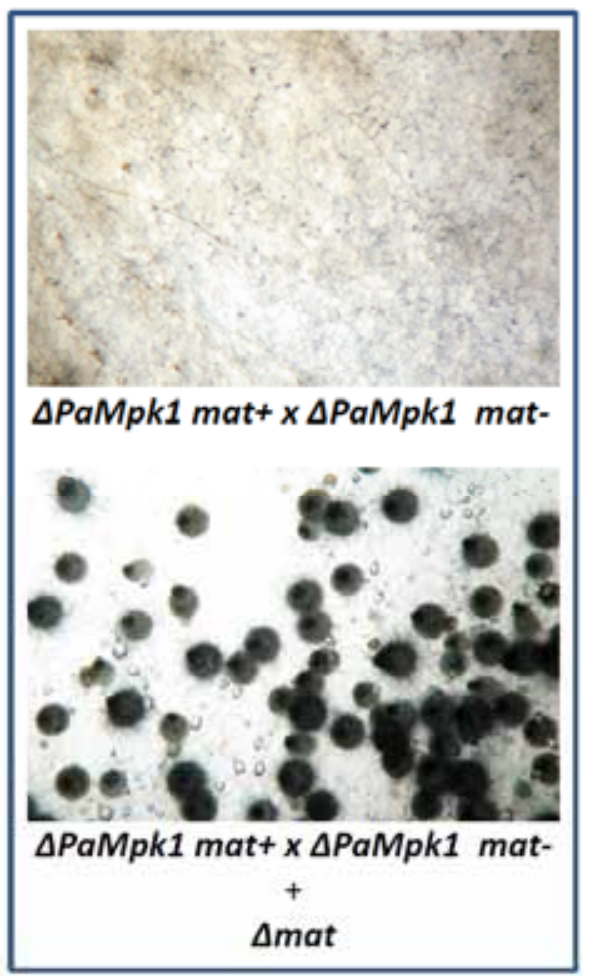

Fig. (5). Mosaic analysis with $\Delta m a t$.

Mosaics were obtained as for Fig. (4). See text for detailed explanation of the outcomes. Right pictures were obtained after seven days of incubation.

incompatible crosses. They tested in such crosses four recessive sexual sporulation mutants, but only to ensure that they could act as both male and female in crosses. However, true mosaic analysis was carried out for the $r c o A \triangle$ mutant of Aspergillus nidulans with blAl [24] to show that the $r c o A$ gene is required in the cleistothecial wall. True mosaic analysis has also been used in Cochliobolus heterotrophus using the albino mutant [25] to study the role of the Mps1 MAP Kinase, which is the orthologue of PaMpk1 in this species. While Mpsl x Mpsl crosses are sterile, Mps 1 x albino ones produce both dark and light pseudothecia. The data can be interpreted as for PaMpk1: Mps 1 is required in the mycelium to promote fruiting body maturation. Note that C. heterostrophus is a member of the Dothideomycetes with an ascolocular development. It is quite notable that the orthologous MAP kinases seem to have a very similar role in such widely divergent species with radically different ontogeny of the fruiting bodies.

\section{MOSAIC ANALYSIS WITH THE AMAT MUTANT}

While grafting and mosaics with autonomous color mutant give valuable information regarding the mycelium $v s$ the fruiting body, they do not give much information regarding the maternal tissues $v s$ the zygotic ones (although the A. nidulans color mutants can be used for such analysis, see [24]). This can be obtained in $P$. anserina thanks to another kind of mosaics, those with $\Delta$ mat mutants. The mat locus controls the mating type determination in $P$. anserina and other heterothallic fungi. In $P$. anserina, deletion of the locus results in strains unable to fertilize or to be fertilized, but that otherwise display a wild-type vegetative phenotype
[26]. Importantly, although $\Delta m a t$ hyphae cannot be present in the zygotic tissues resulting from fertilization, they can be recruited in the maternal ones, especially in the peridium [19]. They can this way rescue mutants that are impaired in their maternal tissues, but not those affected in the zygotic ones. To test this, trikaryons are constructed by mixing together $\Delta m a t$ hyphae with those of the mutant to be tested in both mating types (Fig. 5). If fertility is restored, the gene altered in the mutant is not required in the zygotic tissues, but in the maternal ones. Conversely, perithecia will arrest at a precise stage if the gene is required at this stage in the zygotic tissues. We have used this method to analyze the PaMpk1, PaMpk2, PaNox1, PaNoxR, IDC1 mutants [14, 18, 19] confirming and completing previous data: PaMpkl, PaMpk2 are required only in the mycelium, PaNoxl and PaNoxR only in the maternal tissues of the fruiting bodies and IDCI both in the mycelium and in the maternal tissues. We have also analyzed the $\Delta p a h 5$ beak defect [8] and showed that it was indeed due to a problem in the maternal tissues, as expected since the beak is made from maternal cells and not zygotic ones. Finally, we also studied the $P a A l r 1^{\Delta}$ mutant and showed that the developmental arrest was zygotic, pinpointing a role for this magnesium transporter at a precise stage during maturation inside the ascospores.

\section{CONCLUSION}

The simple methods presented here, combined with more sophisticated genetical (espistasis) and cytological analysis should provide important clues to help unravel the molecular pathways directing development of multicellular fruiting 
bodies in ascomycetes and more generally in fungi. These methods, which were classically used to study animal and plant development, have surprisingly seldom been used in fungi, although they are very easy to implement. Fruiting body mutants are now available for many fungi other than $P$. anserina, including Sordaria macrospora [27], N. crassa [28], A. nidulans [29] and Coprinopsis cinerea (formely Coprinus cinereus) $[30,31]$ and could be analyzed using similar methods. They are complementary to the now more widespread large scale analyses of transcriptomes during fruiting body development as performed in the ascomycetes Fusarium graminearum and Fusarium verticilloides [32-34], N. crassa [35, 36], S. macrospora [37], Pyronema confluens [38], Tuber melanosporum [39-41], Cordyceps militaris [42] and Ophiocordyceps sinensis [43], as well as in the basidiomycetes $C$. cinerea [44], Moniliophthora perniciosa [45], Agrocybe aegerita [46], Lentinula edodes [47, 48], Auricularia polytricha [49] and Ganoderma lucidum [50].

Finally, the methods presented here, especially the mosaics, could be used to revive fertility in some sterile strains, especially those that are used in industrial processes (e.g., Aspergillus niger, Trichoderma reesei, Penicillium chrysogenum, Penicillium roqueforti, etc.), for which prolonged culturing and selection for industrially-efficient strains often results in sterility. Heterokaryosis with fruiting body formation-competent strains inactivated for their mating type and able to provide maternal tissues for fructifications could prove valuable for restoring a complete and efficient sexual cycle in these strains.

\section{CONFLICT OF INTEREST}

The authors confirm that this article content has no conflicts of interest.

\section{ACKNOWLEDGEMENTS}

I thank Dr. Pierre Grognet and Dr. Hervé Lalucque for useful discussion and critical reading of the manuscript.

\section{REFERENCES}

[1] Debuchy R, Berteaux-Lecellier V, Silar P. In: Borkovich K, Ebbole D, Eds. Cellular and Molecular Biology of Filamentous Fungi. Washington DC: ASM Press 2010; pp. 501-35.

[2] Pöggeler S, Nowrousian M, Kück U. In: Kües U, Fischer R, Eds. The Mycota I. Growth, Differentiation and Sexuality. $2^{\text {nd }}$ ed. Berlin, Heidelberg: Springer-Verlag 2006; pp. 325-55.

[3] Silar P. Podospora anserina: from laboratory to biotechnology. In: Horwitz BA, Mukherjee PK, Mukherjee M, Kubicek CP, Eds. Genomics of Soil- and Plant-Associated Fungi. Heidelberg New York Dordrecht London: Springer 2013; pp. 283-309.

[4] Grognet P, Lalucque H, Silar P. The PaAlr1 magnesium transporter is required for ascospore development in Podospora anserina. Fungal Biol 2012; 116: 1111-8.

[5] Espagne E, Lespinet O, Malagnac F, et al. The genome sequence of the model ascomycete fungus Podospora anserina. Genom Biol 2008; 9: R77.

[6] Marcou D, Picard-Bennoun M, Simonet JM. In: O'Brien S, Ed. Genetic Maps. $6^{\text {th }}$ ed. Cold Spring Harbor: Cold Spring Harbor laboratory Press 1982; 3.93-3.101.

[7] Haedens V, Malagnac F, Silar P. Genetic control of an epigenetic cell degeneration syndrome in Podospora anserina. Fungal Genet Biol 2005; 42: 564-77.
[8] Coppin E, Berteaux-Lecellier V, Bidard F, et al. Systematic deletion of homeobox genes in Podospora anserina uncovers their roles in shaping the fruiting body. PLoS One 2012; 7: e37488.

[9] Benkhali JA, Coppin E, Brun S, et al. A network of HMG-box transcription factors regulates sexual cycle in the fungus Podospora anserina. PLoS Genet 2013; 9: e1003642.

[10] Bourne AN, Chiu SW, Moore D. In: Chiu SW, Moore D, Eds. Pattern in fungal development. Cambridge: Cambridge University Press 1996; pp. 126-55.

[11] Silar P. Grafting as a method for studying development in the filamentous fungus Podospora anserina. Fungal Biol 2011; 115: 793-802.

[12] Sellem CH, Marsy S, Boivin A, Lemaire C, Sainsard-Chanet A. A mutation in the gene encoding cytochrome $\mathrm{c} 1$ leads to a decreased ROS content and to a long-lived phenotype in the filamentous fungus Podospora anserina. Fungal Genet Biol 2007; 44: 648-58.

[13] Lalucque H, Silar P. In vivo labelling of functional ribosomes reveals spatial regulation during starvation in Podospora anserina. BMC Genet 2000; 1: 3.

[14] Lalucque H, Malagnac F, Brun S, Kicka S, Silar P. A nonmendelian MAPK-generated hereditary unit controlled by a second MAPK pathway in Podospora anserina. Genetics 2012; 191: 41933.

[15] Beckett A, Wilson IM. Ascus cytology of Podospora anserina. J Gen Microbiol 1968; 53: 81-7.

[16] Malagnac F, Lalucque H, Lepere G, Silar P. Two NADPH oxidase isoforms are required for sexual reproduction and ascospore germination in the filamentous fungus Podospora anserina. Fungal Genet Biol 2004; 41: 982-97.

[17] Coppin E, Silar P. Identification of PaPKS1, a polyketide synthase involved in melanin formation and its use as a genetic tool in Podospora anserina. Mycol Res 2007; 111: 901-8.

[18] Brun S, Malagnac F, Bidard F, Lalucque H, Silar P. Functions and regulation of the Nox family in the filamentous fungus Podospora anserina: a new role in cellulose degradation. Mol Microbiol 2009; 74: 480-96.

[19] Jamet-Vierny C, Debuchy R, Prigent M, Silar P. IDC1, a Pezizomycotina-specific gene that belongs to the PaMpk1 MAP kinase transduction cascade of the filamentous fungus Podospora anserina. Fungal Genet Biol 2007; 44: 1219-30.

[20] Johnson TE. Analysis of pattern formation in Neurospora perithecial development using genetic mosaics. Dev Biol 1976; 54: 23-36.

[21] Zonneveld BJM. Morphology of initials and number of nuclei intiating cleistothecia in Aspergillus nidulans. Trans Br Mycol Soc 1988; 90: 365-73.

[22] Apirion D. Formal and physiological genetics of ascospore colour in Aspergillus nidulans. Genet Res (Camb) 1963; 4: 276-83.

[23] Bruggeman J, Debets AJ, Swart K, Hoekstra RF. Male and female roles in crosses of Aspergillus nidulans as revealed by vegetatively incompatible parents. Fungal Genet Biol 2003; 39: 136-41.

[24] Todd RB, Hynes MJ, Andrianopoulos A. The Aspergillus nidulans rcoA gene is required for veA-dependent sexual development. Genetics 2006; 174: 1685-8.

[25] Igbaria A, Lev S, Rose MS, et al. Distinct and combined roles of the MAP kinases of Cochliobolus heterostrophus in virulence and stress responses. Mol Plant Microbe Interact 2008; 21: 769-80.

[26] Coppin E, Arnaise S, Contamine V, Picard M. Deletion of the mating-type sequences in Podospora anserina abolishes mating without affecting vegetative functions and sexual differentiation. Mol Gen Genet 1993; 241: 409-14.

[27] Engh I, Nowrousian M, Kuck U. Sordaria macrospora, a model organism to study fungal cellular development. Eur J Cell Biol 2010; 89: 864-72.

[28] Colot HV, Park G, Turner GE, et al. A high-throughput gene knockout procedure for Neurospora reveals functions for multiple transcription factors. Proc Natl Acad Sci USA 2006; 103: 10352-7.

[29] Han KH. Molecular genetics of Emericella nidulans sexual development. Mycobiology 2009; 37: 171-82.

[30] Kamada T. Molecular genetics of sexual development in the mushroom Coprinus cinereus. Bioessays 2002; 24: 449-59.

[31] Kues U. Life history and developmental processes in the basidiomycete Coprinus cinereus. Microbiol Mol Biol Rev 2000; 64: $316-53$. 
[32] Hallen HE, Huebner M, Shiu SH, Guldener U, Trail F. Gene expression shifts during perithecium development in Gibberella zeae (anamorph Fusarium graminearum), with particular emphasis on ion transport proteins. Fungal Genet Biol 2007; 44: 1146-56.

[33] Sikhakolli UR, Lopez-Giraldez F, Li N, Common R, Townsend JP, Trail F. Transcriptome analyses during fruiting body formation in Fusarium graminearum and Fusarium verticillioides reflect species life history and ecology. Fungal Genet Biol 2012; 49: 663-73.

[34] Qi W, Kwon C, Trail F. Microarray analysis of transcript accumulation during perithecium development in the filamentous fungus Gibberella zeae (anamorph Fusarium graminearum). Mol Genet Genomics 2006; 276: 87-100.

[35] Wang Z, Lopez-Giraldez F, Lehr N, et al. Global gene expression and focused knockout analysis reveals genes associated with fungal fruiting body development in Neurospora crassa. Eukaryot Cell 2014; 13: 154-69.

[36] Wang Z, Lehr N, Trail F, Townsend JP. Differential impact of nutrition on developmental and metabolic gene expression during fruiting body development in Neurospora crassa. Fungal Genet Biol 2012; 49: 405-13.

[37] Gesing S, Schindler D, Nowrousian M. Suppression subtractive hybridization and comparative expression analysis to identify developmentally regulated genes in filamentous fungi. J Basic Microbiol 2013; 53: 742-51.

[38] Traeger S, Altegoer F, Freitag $\mathrm{M}$, et al. The genome and development-dependent transcriptomes of Pyronema confluens: a window into fungal evolution. PLoS Genet 2013; 9: e1003820.

[39] Ceccaroli P, Buffalini M, Saltarelli R, et al. Genomic profiling of carbohydrate metabolism in the ectomycorrhizal fungus Tuber melanosporum. New Phytol 2011; 189: 751-64.

[40] Montanini B, Levati E, Bolchi A, et al. Genome-wide search and functional identification of transcription factors in the mycorrhizal fungus Tuber melanosporum. New Phytol 2011; 189: 736-50.

[41] Tisserant E, Da Silva C, Kohler A, Morin E, Wincker P, Martin F. Deep RNA sequencing improved the structural annotation of the
Tuber melanosporum transcriptome. New Phytol 2011; 189: 88391.

[42] Yin Y, Yu G, Chen Y, Jiang S, Wang M, Jin Y, et al. Genomewide transcriptome and proteome analysis on different developmental stages of Cordyceps militaris. PLoS One 2012; 7: e51853.

[43] Xiang L, Li Y, Zhu Y, et al. Transcriptome analysis of the Ophiocordyceps sinensis fruiting body reveals putative genes involved in fruiting body development and cordycepin biosynthesis. Genomics 2014; 103: 154-9.

[44] Cheng CK, Au CH, Wilke SK, et al. 5'-serial analysis of gene expression studies reveal a transcriptomic switch during fruiting body development in Coprinopsis cinerea. BMC Genomics 2013; 14: 195.

[45] Pires AB, Gramacho KP, Silva DC, et al. Early development of Moniliophthora perniciosa basidiomata and developmentally regulated genes. BMC Microbiol 2009; 9: 158.

[46] Wang M, Gu B, Huang J, et al. Transcriptome and proteome exploration to provide a resource for the study of Agrocybe aegerita. PLoS One 2013; 8: e56686.

[47] Chum WW, Ng KT, Shih RS, Au CH, Kwan HS. Gene expression studies of the dikaryotic mycelium and primordium of Lentinula edodes by serial analysis of gene expression. Mycol Res 2008; 112: 950-64.

[48] Zhong $\mathrm{M}$, Liu B, Wang $\mathrm{X}$, et al. De novo characterization of Lentinula edodes $\mathrm{C}(91-3)$ transcriptome by deep Solexa sequencing. Biochem Biophys Res Commun 2013; 431: 111-5.

[49] Zhou Y, Chen L, Fan X, Bian Y. De novo assembly of Auricularia polytricha transcriptome using illumina sequencing for gene discovery and SSR marker identification. PLoS One 2014; 9: e91740.

[50] Yu GJ, Wang M, Huang J, et al. Deep insight into the Ganoderma lucidum by comprehensive analysis of its transcriptome. PLoS One 2012; 7: e44031.

(C) Philippe Silar; Licensee Bentham Open.

This is an open access article licensed under the terms of the Creative Commons Attribution Non-Commercial License (http://creativecommons.org/ licenses/by-nc/3.0/), which permits unrestricted, non-commercial use, distribution and reproduction in any medium, provided the work is properly cited 